University for Business and Technology in Kosovo

UBT Knowledge Center

Oct 27th, 1:30 PM - 3:00 PM

\title{
An Approach to The Capacity Management of the Public Health Institutions: University Clinical Centre of Kosova Case Study
}

\author{
Ylber Limani \\ University for Business and Technology, ylber.limani@ubt-uni.net \\ Marigona Markaj \\ University for Business and Technology, mm32858@ubt-uni.net
}

Follow this and additional works at: https://knowledgecenter.ubt-uni.net/conference

Part of the Business Commons

\section{Recommended Citation \\ Limani, Ylber and Markaj, Marigona, "An Approach to The Capacity Management of the Public Health Institutions: University Clinical Centre of Kosova Case Study" (2018). UBT International Conference. 276. https://knowledgecenter.ubt-uni.net/conference/2018/all-events/276}

This Event is brought to you for free and open access by the Publication and Journals at UBT Knowledge Center. It has been accepted for inclusion in UBT International Conference by an authorized administrator of UBT Knowledge Center. For more information, please contact knowledge.center@ubt-uni.net. 


\title{
An Approach to The Capacity Management of the Public Health Institutions: University Clinical Centre of Kosova (UCCK) Case Study
}

\author{
Ylber Limani ${ }^{1}$, Marigona Markaj ${ }^{2}$ \\ ${ }^{1,2}$ UBT College, Prishtina \\ ylber.limani,.mm32858\{@ubt-uni.net\}
}

\begin{abstract}
This research aims the evaluation of the state of capacity management condition in the public and private health enterprises, as well as its impact on the overall performance, on the quality of services provided, and on achieving enterprise strategic objectives. The supposition is that enterprise capacity in health sector face deficiency on strategic planning. The research model includes a combination of qualitative and quantitative methods in order to examine the problem more intensely. The research is conducted by interviewing respondents engaged in health public and private enterprises management. Capacity management has to do capacity design, planning and organizing, as well as establishing monitoring mechanisms for its control. Thought, enterprises intend to utilize their resources optimally in order to achieve its strategic goals, objectives and better performance in general. In order to achieve this, healthcare enterprises in the country should be focused on strategic planning with distinct focus on capacity management. The research results provide with the conceptual model for more effective capacity management in the health sector, among findings from the explorative research
\end{abstract}

Keywords: Capacity management, healthcare, strategic planning, services, performance. 


\section{Introduction}

Among the key challenges within the health sector in general is the improvement of the services provided and the cost reduction. Apart from representing challenges in one side, they represent strategic objectives toward the achievement of main strategic goal. The purpose of this study is to assess the importance of capacity management in public and private health enterprises, as well as its impact on overall performance of health companies, in the quality of services provided and in achieving their strategic objectives. The study specifically focuses on the capacity management at the University Clinical Center of Kosova (UCCK), giving an overview of organizations various sectors and their capability to address and satisfy the demand.

The methods utilized in this research fluctuates from qualitative to quantitative depending on the study objectives. Thoroughly, paper has been developed based on the review of contemporary literature, scientific articles, interviews and surveys conducted in the health sector: including public and private healthcare service providers.

The position of this work attitudes in the role and importance of capacity management in achieving the strategic objectives of public and private health enterprises in improving the quality of services and effectively managing the cost.

The purpose of the study is achieved through following objectives:

- Deliverance of an analytical overview of the state of affairs in the health sector in the country;

- Assessment of the importance of capacity management in public and private health enterprises and its impact on organizations performance;

- Establishment of recommendations on formulating strategies for capacity management in health sector.

The research questions of this paper addressed important issues, and they are composed as follows:

How is the state of affairs regarding existing formal strategies, capacity management and overall hospital performance?

How is capacity managed in health organizations in Kosovo and what can be improved in this regard?

How can affect proper capacity management in improving the quality of services provided by health institutions in Kosovo?

The empirical analysis of the data obtained from the surveys and interviews conducted in the health sector, and the interpretation of results, were realized through qualitative, quantitative and comparative methods, as well as the combination of the theoretical part with the practical part of the research. The use of shared software program has facilitated the analysis and made it more comprehensible.

This paper is considered to be of scientific and practical importance, as it is one of the creative and different on this specific field, which discusses the importance of capacity management and the implementation of strategies for achieving the objectives of health enterprises. 


\section{Literature review}

This study addresses capacity management issues which mainly relate to the utilization of the resources of the health institution: staff, space, and the utilization of technologies and inventory. In this esteem, the literature examination is directed towards the three identified and well-defined factors.

The literature review is conducted on the attention of main topics concerned with the capacity management such as: capacity management as a science, measuring enterprise capacity and capacity planning, capacity planning process, capacity management in public enterprises, capacity strategy, capacity management in healthcare institutions, management of public health institutions in Kosovo as a case study.

The capacity relates to the maximum level of enterprise operations that are carried out over a certain period of time under normal working conditions (Rahabok, 2016).

The capacity is related to the maximum level of enterprise operations are capable of carrying out over a certain period of time under regular working conditions (Slack, et al, 2013). It can also be defined as the ability of a producer or service provider to accomplish the objectives of the organization over a given period of time and is considered as an integral component of the investment process (Stock et al, 2017).

Nonetheless, in capacity management different authors agree that capacity management provides more quality services and significant reduction in the cost structure (Dingman, et al, 2012; Slack et al, 2016; Lee et al, 2012). Though, according to other group of authors, the disagreement stems from the fact that capacity management should be linked to the organization's strategic goals and its main objectives. It states here that if an enterprise intends to reduce the cost structure, it will affect the services provided and if it is intended to differentiate services, then the enterprise finds it difficult to pursue a cost-cutting and resource optimization strategy, as more time and resources are needed if the provision of more differentiated services is sought (Smith-Daniels et al, 1988, Hitt et al, 2011).

In capacity management as a science is also stated monitoring and capacity control. In order to know how the company's resources are being used, how services are performed and how capacity is managed in general, that all this information is taken from monitoring and controlling the capacity processes.

Capacity planning in an organization can be carried out on two levels, which correspond to long-term and short-term decisions. The first level of capacity decisions is strategic and long-term. It is of great importance to the organization to plan and control the process of determining the amount of output that can be achieved by the manufacturing / service unit (Williams \& Hall, 2010).

Capacity measurements through inputs and outputs are real indicators of capacity utilization of the enterprise as well as important data for the formulation of strategies on capacity management. Capacity measurement is an overview of the Enterprise on the allocation of its resources, their level of utilization and provides important information on the enterprise's capability (David, 2011; Slack et al, 2013). 
Table 1. Measurement examples of input and output capacity in different industries

\begin{tabular}{lll}
\hline Type of business & Capacity input measurement & Capacity output measurement \\
\hline $\begin{array}{l}\text { Hospital } \\
\text { Manufacturing vehicles }\end{array}$ & $\begin{array}{l}\text { Beds available for months } \\
\text { Hours of living work and } \\
\text { machine work }\end{array}$ & $\begin{array}{l}\text { Number of patients per month } \\
\text { Cars for turn }\end{array}$ \\
$\begin{array}{l}\text { Pizzeria } \\
\text { Ice cream maker }\end{array}$ & $\begin{array}{l}\text { Hours of operation per day } \\
\text { Retail store }\end{array}$ & $\begin{array}{l}\text { Kumber of pizzas per day } \\
\text { day }\end{array}$ \\
\hline
\end{tabular}

Source: David, 2011

Capacity measurements through inputs and outputs are real indicators of capacity utilization of the enterprise as well as important data for the formulation of its capacity management strategies. Capacity measurement is an outline of the enterprise concerned with its resource allocation, level of exploitation, and provides important information on the enterprise's ability to cope with demand fluctuations and its affinity for flexibility in order to fit these requirements (David, 2011).

Capacity management in healthcare institutions

Health care resources are generally becoming limited and expensive, while providing a qualitative service to patients. Therefore, it's important that the capacity be designed in such a way that resources are optimized and used in the most efficient way, by increasing the percentage of resource utilization and shortening the cost. Thought, the basic problem of organizational decision-making, both in the public and private sectors is the achievement of a balance in the resource allocation program, which will provide a readiness system in the short, medium and long term. Achieving this objective requires flexibility in confronting a wide range of competitive actions (Steiss, 2003).

Therefore, an important thing for employees in the public services sector is concerned with the question how to allocate resources between competing demands. They decide over humble questions: who to provide with service and how to provide the service? Allocation of resources is the main issue of public services and has always produced difficult situations (Fisher, 2002). Resource allocation problems can be reduced or eliminated if a public organization has a sound, comprehensive strategic plan, if there is a service delivery strategy, accurate demand planning and capacity management through the optimum utilization of its resources (Steiss, 2003; Van Houdenhoven, et al, 2012).

The health sector today mainly faces two problems which have to do with increasing the quality of services as well as reducing the overall costs of hospitals and clinics. Therefore, the health institutions need appropriate and explicit health care strategy (Green, 2005; Dingman et al, 2012).

It is important that the health care strategy be comprehensive and consistent with the objectives of the organization. Among other things, it is important that the strategy on capacity management comprise of these objectives: 
- To provide services at the right level for each patient,

- To allow for more staffing,

- To increase the satisfaction of staff, doctors and patients, and

- Reduce operational costs (Dingman, et al, 2012).

The availability of a well-formulated strategy by the healthcare institution and the harmonization of operations and this strategy with the goals of the organization are vital for the institution to operate efficiently and effectively (Hitt et al, 2011), increasing incomes, providing quality services and enhancing the satisfaction of its stakeholders (Lee, et al, 2012).

\section{Operations strategy}

Healthcare institutions should possess and implement operations strategies through which the vision and objectives of that institution are achieved and accomplished. Operations strategy and operations control impact increasing the quality of service delivery and capacity management (Hitt et al, 2011). The operations strategy determines the allocation of resources of the organization, as well as optimizing their use, maximizing their utilization, and reducing operating costs (Lee, et al, 2012). Correspondingly, capacity management is more efficient and effective in maximizing the use of health care resources, this maximization applies both to human resources, space, technology, and material resources.

The entire transformation process refers to the value chain of the health institution, from the creation of the service up to its offering to the last patient. This full process should provide the institution with the conditions for value creation (David, 2011), which is translated into satisfied patients based on the highest level of service and benefits the reputation of the health institution in general. In the future this value can be transformed into a brand that reflects good healthcare services for customers (Kotler \& Armstrong, 2013).

Health institutions, both, public and private, should own well-formulated strategies on answering many critical issues related to the way the provide their services (Dingman, et al, 2012). Because public health delivers the clients very sensitive services, it is of particular importance that the services are adequate and qualitative. Therefore, it is of particular importance for the healthcare institutions to have specific strategies on service delivery and customer care so that the quality of the services provided is as high as the customers feel secure in obtaining the service (Cardoen, et al, 2010).

Principles of strategic management in health care institutions are: patients first; safety; personal responsibility; defined authority; clear responsibility; leadership; multidisciplinary work; support performance; open culture; continuous quality improvement (Unit, 2015).

In conclusion, the literature findings argue that capacity management has very important role for the regular functioning of the healthcare institutions. In addition, capacity management ensures supplementary confident performance making all stakeholders satisfied with the services provided. 


\section{Case Study: UCCK}

The capacity assets of a UCCK healthcare institution are mainly focused on two categories: number of available beds and number of professional staffs, and assisting staff. Throughout these two assets, usually the needs and requirements of the patients are managed. The capacity of the health care institution usually become managed through the function of demand planning. In this case UCCK's capacity, is made-toorder depending on demands and needs of the clients. In these circumstances, capacity management is defined as management of the readiness of the institution in meeting the requirements of the clients through the optimal use of resources.

In healthcare institutions, specifically at UCCK, usually capacity measurements are conducted based on beds available and staff. From data acquired directly from the survey and statistical reports, has been noticed that UCCK has a large capacity of service delivery, although, the demand for the services of public institutions is always higher than in the private sector. The UCCK has a total of 2,015 beds, 1,050 doctors and 1,704 nurses through these resources and with this capacity the institution tends to manage the demands of patients from all around the country the Republic of Kosovo. Through its strategies and demand planning, UCCK adjusts its capacity to provide better quality services to patients, while also trying to reduce delays in their treatments. It is important for UCCK to allocate its resources based on the requirements of clinics and sectors. Above and beyond allocating resources, it should also organize its material and human resources in such a way that they can use them efficiently and effectively, affecting the optimization of the capacity and the fulfilment of the patients' requirements with the most possible quality services. Capacity management has a direct influence on the demand management and the delivery of quality services. Therefore, UCCK tends to manage its capacity through its strategies maintaining the provision of quality services at the lowest cost.

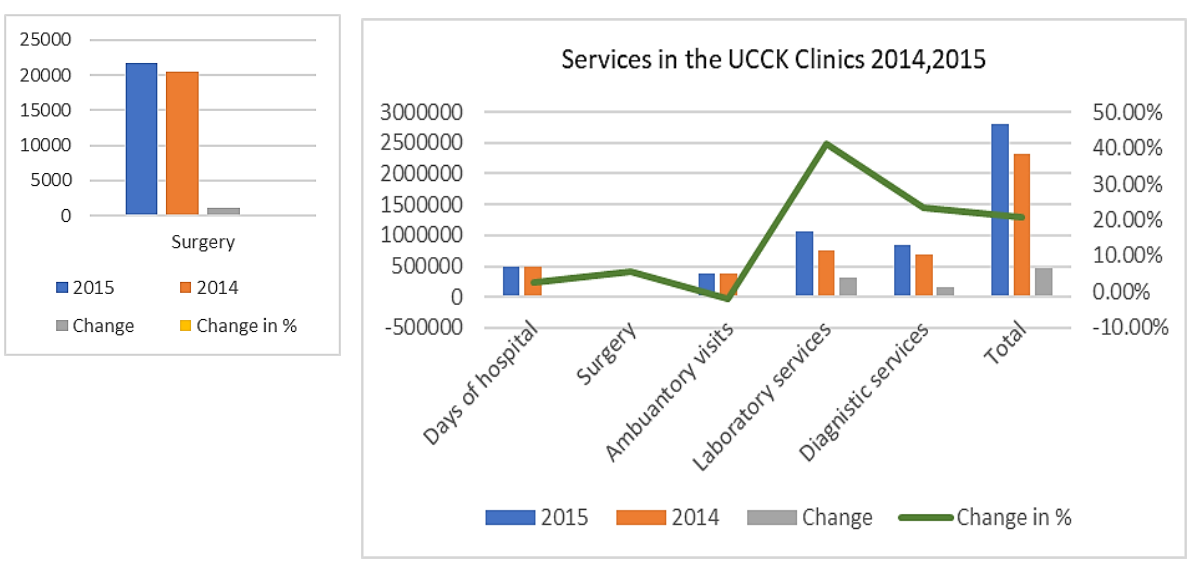

Fig.1. Services at UCCK clinics in 2014 and in 201 (Source: RKS Government, 2015) 


\section{Problem statement and research questions}

The health sector currently faces primarily two problems, which concern the quality level of services as well as the reduction of overall hospital and clinic spending. The study will address capacity management issues, which mainly relate to the use of capacity constitution components such as healthcare resources, staff, space and the use of modern technologies. The structural components the organizations capacity signifies the organizations capability on improving the quality of services and managing the cost flow over time long.

Pointing to the state of the health sector in Kosovo, with particular emphasis on UCCK, respectively the findings displayed in the section: Case study, the research resulted to a proposal for model development which potentially could be used as a basement for strategy development concerned with capacity management in health sector in order to improve the quality of services and the cost flow. This research results also serve as input for future studies on developing efficient techniques and models for capacity management in health sector and providing fundamentals for new approaches in this respect.

I accordance of addressing the problem identified, the following three research questions are elevated and answered in this research:

1. How is the state of affairs regarding existing formal strategies, capacity management and overall hospital performance?

2. How is capacity managed in health organizations in Kosovo and what can be improved in this regard?

3. How can affect proper capacity management in improving the quality of services provided by health institutions in Kosovo? 


\section{The methodology}

Research sample and design: This study consists of empirical research which is illustrated through statistical reports and surveys, as well as the qualitative part which was conducted through interviews in hospitals and UCCK. The project of conducting interviews and surveys has included the health sector, respectively UCCK. The quantity of researched health-care institutions is considered to be representative sample that reflects current strategies, capacity and developments in the health sector in Kosovo.

Data Collection: Resources used include primary and secondary resources. Secondary data sources are derived from contemporary literature of strategic management, operations and other relevant fields, as well as articles from various scientific journals. While the primary data collection was conducted in the field through interviews and surveys in the Kosovo health sector, namely in the city of Prishtina. Surveys and interviews are based on the semi-structured questionnaire.

The questionnaire contains a total of 15 questions, these questions created and developed in harmony with the goals of the paperwork.

Samples: the high-level managers and mid-level managers of the various UCCK sectors have been sampled, so that the work is enriched with the data making possible a multidimensional approach to the problem. While through research questions the study is given a multidimensional aspect of the current state of the health sector in

The case study conducted in this research is related to Kosovo as a developing and in transition country, with particular emphasis on UCCK, strategies on capacity management and improvement of the current quality of services in hospitals and clinics, cost structure and the performance of health institutions in general.

The data obtained from the interviews were elaborated and analyzed through combinations of the theoretical fragment with the practical fragment. The theoretical and empirical analysis are conducted, accompanied with comparative method. Findings display the proportions, similarities and differences between the strategies of the health enterprises and their state of matters rapport at competition level. 


\section{Results}

The results of this study maintain the fundamental assumptions signifying that a public or private health institution can benefit a significant portion from effective capacity management.

With the current state of staff, beds available and technical-technological equipment, health units in Kosovo do not meet the needs of patients at the appropriate level.

According to the research results, also supported by the qualitative research, it is important that demand develop planned in advance. This will create solid circumstances for reasonable and effective investment in capacity assets. The operative capacity management provides the health institutions with the required flexibility and speed to meet the demand adequately. At operational level, effective capacity management affects the improvement of services and reduces the waiting time of patients, as well as the recovers the cost and benefits ration in of the clinics.

Throughout the survey we encountered in the absence of modern technology at UCCK. In this regard the study discovers that $36 \%$ of respondents think they need new technology in their clinics. In the cases where new technology is implemented, the number of treated patients increases $89.3 \%$ according to respondents.

The biggest problem encountered in the use of technology in the clinic, has to do with the lack of adequate staff for use and for maintenance. This statistic is produced based on the answers of $42.9 \%$ of respondents. In this regard, another $42.9 \%$ of the respondents say that raising the staff, space and technology qualitatively and quantitatively will meet the requirements for providing modern and sufficient medical services.

First finding of this research provides with the answer on question concerned with the strategy. In Kosovo, there is a lack of formal capacity management strategies, which in turn affects the cost structure and the quality of service delivery. Consequently, the resources, both human and material, are not optimally utilized. Apart from the absence of a formal strategy on capacity management, it is noticed that the general strategy of the hospital show lack on the harmonization of the strategic objectives of the hospital and the practises of using its capacity.

The answer on the second question of this research indicates that assets in the clinics do not suffice and they should be increased approximately $35 \%$ to $40 \%$. In that way capacity utilization will become more optimistic and will provide with the possibilities of quality of service delivery improvement.

Finally, the research recommends a project proposal for hospital capacity management. This project aims to propose a technical and practical plan on capacity management in a particular clinic. This plan proposes the clinics to focus mainly on 4 pillars:

- Re-designing the strategic objectives of the clinic and outlining the the capacity management objectives within the strategy of the clinic,

- Development of effective forecasting techniques related to the potential patients' flow;

- Developing and implementing the monitoring system of capacity assets and increasing their flexibility;

- Developing and implementing the service performance evaluation system. 


\section{Conclusions and Recommendations}

\section{Conclusions}

Health enterprises in the process of strategy implementation need to develop and implement a mechanism for monitoring and controlling such implementation.

Investing in smart capacity management assets in general is the best strategy to meet patients' demands and in the long run to reduce costs.

Hospitals and medical centres in Kosovo, in line with the strategic objectives of the Ministry of Health are increasing the staff and improving the infrastructure. Nonetheless, the amount of endeavours in that direction is not sufficient and deeds upgrading.

With the current staff status, hospital inventory, and technical-technological equipment, health units in Kosovo do not meet the needs of patients at the appropriate level, both quantitatively and qualitatively

\section{Recommendations}

Forecasting demand and capacity management plans should be consistently harmonized in order to ensure an optimal use of resources and to treat patients at a satisfactory level, making them satisfied with their services.

Hospital management should be proactive and flexible to change and to adopt this modern approach to strategy formulation.

Management should also influence the creation of an organizational culture that considers staff to be the most important asset in the organization, respectively in the hospital.

The capacity management plan should be flexible in order to adapt to the changes and flows of the patients. This flexibility ensures that the quality of the services provided remains at the right level. 


\section{References}

Rahabok, I. (2016). VMware performance and capacity management. Packt Publishing. Braithwaite,

Cardoen, Demeulemeester, E. \& Belien, J. (2010). Operating room planning and scheduling: A literature review. European Journal of Operational Research, pp. 921-932.

David, F. R. (2011) Strategic Management: Concepts and cases. 13th edition Hrsg. New Yersey: Prentice Hall.

Dingman, B., Theobald, B. \& Jefferson, J. (2012). Capacity Strategy: The science of improving future performance, s.l.: GE Healthcare.

EW, H., Van Houdenhoven, M. \& Hulshof, P. (2012) A framework for healthcare planning and control: Handbook of healthcare system scheduling. s.1.:Springer .

Fisher, C. (2002) Resource allocation in the public sector. London: Routledge.

Green, L. V., 2005. Capacity Planning and Management in Hospitals. Columbia: Columbia University.

Hitt, M. A., Duane, I. \& Hoskisson R. (2011) Strategic Management: Competetiveness \& Globalization. 9th Edition Hrsg. USA: South-Western Cengage Learning.

Kotler, P. \& Armstrong, G. (2013) Marketing Principles. 15th Edition, Prentice Hall.

Slack, N., Brandon-Jones, A. \& Johnston, R., (2013) Operations Management. Harlow: Pearson Education Limited.

Smith-Daniels, V. L., Smith-Daniels, D. E. \& Schweikhart, S. B. (1988) Capacity Management in Health Care Services: Review and Future Research Directions. Decision Sciences, Issue 4, p. 889-919.

Stock, J., Cantara, M., Flaherty, J. \& Venkataraman, R. (2017). Capacity management: A key risk measure. Canada: White Papaer Series.

Unit, S. D., (2015). Technical Guidance Introducing Demand and Capacity Planning, Dublin: Special Delivery Unit, Health Service Executive.

Williams, A. M. \& Hall, M. (2010) Tourism and migration: New relationships between production and consumption. An International Journal of Tourism Space, Place and Environment, pp. 5-27.

Slack, N., Brandon-Jones, A. and Johnston, R. (2016). Operations management. $8^{\text {th }}$ edition. Pearson

RKS Government, 2016. Health statistics report. [Online] http://ask.rksgov.net/media/2470/statistikat-e-shendetesis-2015.pdf 